\title{
Matrix Models, Quantum Gravity and the Spectral Dimension
}

\section{Fermín Viniegra*, Mike Peardon and Sinéad M. Ryan}

Trinity College Dublin

E-mail: viniegra@maths.tcd.ie

Monte Carlo studies on non-perturbative 2D Euclidean Quantum Gravity can be done with the use of the Dynamical Triangulations method. The Monte Carlo moves defined within the dynamical triangulation method can be encompassed into a Random one-Matrix Model. In this project the Spectral Dimension associated to the random matrix model is analysed.

XXIIIrd International Symposium on Lattice Field Theory

25-30 July 2005

Trinity College, Dublin, Ireland

*Speaker. 


\section{Introduction}

The structure of spacetime is one of the most important concepts the theory of quantum gravity aims to understand. In recent years it has been shown that spacetime may exhibit a fractal structure at microscopic scales $[1,2,3]$. When describing a fractal structure, many different definitions of dimension emerge [4], among them is the Intrinsic Hausdorff Dimension or Fractal Dimension $d_{H}$, Extrinsic Hausdorff Dimension $D_{H}$ and the Spectral Dimension $d_{S}$ [5].

There are several well-known numerical methods to describe quantum gravity (see for example [6]). One of the most important numerical models in 2D quantum gravity is derived from the dynamically triangulated random surface model $[7,8]$ in which summations over piecewise linear surfaces are used to define reparametrization-invariant forms of the observable quantities [9].

One very attractive aspect of the dynamical triangulation model is that the ergodic moves in the set of triangulations can be encoded in a simple random matrix model [10]. In this model the main quantity to measure is, of course, the eigenvalue density which in the planar limit is directly related to the expectation values of the theory.

Our objective in this project is to investigate further the properties of the spectral dimension in the matrix model context.

In the next sections we briefly review some concepts needed from quantum gravity to calculate the spectral dimension. Next we review some topics on random matrix models and in the last section we outline the main point to cover in our project.

\section{Two Dimensional Quantum Gravity}

The starting point is the partition function of two-dimensional euclidean quantum gravity where each member of the ensemble is a manifold $M$ of dimension two, closed, compact, connected, orientable and of genus $g$

$$
Z(\Lambda ; G, \lambda)=\sum_{\mathbf{g} \geq 0} \int \mathscr{D}\left[g_{a b}\right] e^{-S_{E H}[g ; G, \Lambda]} \int \mathscr{D} \phi e^{-S_{M}[g, \phi ; \lambda]} .
$$

The measure $\mathscr{D}\left[g_{a b}\right]$ is over all diffeomorphism classes $\left[g_{a b}\right]$ of metrics $g_{a b}$ on the manifold $M, G$ is the gravitational constant, $\phi$ represents the matter fields with $\lambda$ the parameters of the theory and $S_{M}$ the matter action, while $S_{E H}$ is the Einstein-Hilbert action.

In two dimensions the Einstein-Hilbert action, the integral of the Ricci Scalar, is a topological invariant, $\chi(\mathrm{g})=\int_{M} d^{2} x \sqrt{g} R=2-2 \mathrm{~g}$ which separates the genera as

$$
Z(\Lambda ; G, \lambda)=\sum_{\mathrm{g} \geq 0} e^{\frac{\chi(\mathrm{g})}{G}} Z(\Lambda ; \lambda) . \quad \text { where } \quad Z(\Lambda ; \lambda)=\int \mathscr{D}\left[g_{a b}\right] e^{-\Lambda \int_{M} d^{2} x \sqrt{g}} \int \mathscr{D} \phi e^{-S_{M}[g, \phi ; \lambda]}
$$

It is often convenient to separate the second equation in (2.2) into canonical and grand canonical partition functions. The canonical partition function is defined to be the ensemble of manifolds $M$ with fixed volume

$$
Z(V ; \lambda)=\int \mathscr{D}\left[g_{a b}\right]_{V} \int \mathscr{D} \phi e^{-S_{M}[g, \phi ; \lambda]} \quad \text { where } \quad \mathscr{D}\left[g_{a b}\right]_{V}=\mathscr{D}\left[g_{a b}\right] \delta\left(\int_{M} d^{2} x \sqrt{g}-V,\right)
$$


which allows us to recover the second equation in (2.2) by using a Laplace transform that defines the grand canonical partition function

$$
Z(\Lambda ; \lambda)=\int_{0}^{\infty} d V e^{-\Lambda V} Z(V ; \lambda) .
$$

The expectation values on the canonical and grand canonical ensembles are defined in the usual way. The next step is to define a reparametrization-invariant form of the two-point correlation function for the canonical ensemble

$$
\begin{aligned}
G_{G C E}(\Lambda, r) & =\int \mathscr{D}\left[g_{a b}\right] e^{-\Lambda \int_{M} d^{2} x \sqrt{g}} \int_{M} d^{2} x \sqrt{g(x)} \int_{M} d^{2} x^{\prime} \sqrt{g\left(x^{\prime}\right)} \delta\left(d_{g}\left(x, x^{\prime}\right)-r\right) \\
G_{C E}(V, r) & =\int \mathscr{D}\left[g_{a b}\right]_{V} \int_{M} d^{2} x \sqrt{g(x)} \int_{M} d^{2} x^{\prime} \sqrt{g\left(x^{\prime}\right)} \delta\left(d_{g}\left(x, x^{\prime}\right)-r\right),
\end{aligned}
$$

where $d_{g}\left(x, x^{\prime}\right)$ represents the geodesic distance between two points in spacetime and where both equations are connected by a Laplace equation similar to (2.4).

\section{Spectral Dimension}

The spectral dimension can be obtained in several ways, the simplest way is by analysing the return probability of a system in a diffusion process. The main idea is to use a collection of freely propagating particles to probe the structure of spacetime. Given a two-dimensional manifold, $M$ with a metric $g_{a b}(x)$, we can define a diffusion process on this manifold by introducing a step parameter, called the diffusion parameter $\sigma$ and the wave function $\psi(x, \sigma)$. The diffusion process is governed by the equation

$$
\frac{\partial}{\partial \sigma} \psi(x, \sigma)=\Delta_{g} \psi(x, \sigma)
$$

where $\Delta_{g}=\frac{1}{\sqrt{g}} \partial_{a} \sqrt{g} g^{a b} \partial_{b}$ is the Laplace-Beltrami operator corresponding to the metric $g_{a b}$. It is assumed the system is initially peaked at the point $x_{0}$ when $\sigma=0$

$$
\psi(x, 0)=\frac{\delta\left(x-x_{0}\right)}{\sqrt{g(x)}}
$$

Related to the inverse of the Laplace-Beltrami operator is the probability distribution for diffusion on a compact manifold $M$ with metric $g_{a b}$, the heat kernel, $K\left(x, x^{\prime} ; \sigma\right)$ which can be used to express the formal solution to the diffusion equation

$$
\psi(x, \sigma)=\int_{M} d^{2} x^{\prime} \sqrt{g} K^{\prime}\left(x, x^{\prime} ; \sigma\right) \psi\left(x^{\prime}, 0\right) .
$$

From general considerations it has been shown [] that $K\left(x, x^{\prime} ; \sigma\right)$ has the asymptotic short distance expansion

$$
K\left(x, x^{\prime} ; \sigma\right) \sim \frac{e^{-d_{g}\left(x, x^{\prime}\right) / 4 \sigma}}{4 \pi \sigma^{d / 2}}, \sum_{i=0}^{\infty} \sigma^{n} a_{n}\left(x, x^{\prime}\right)
$$

where the coefficients $a_{n}$ are reparametrization invariant. The last equation allows us to define the spectral dimension by calculating the average probability distribution for the system to return to its initial state after $\sigma$ steps in the quantum gravity canonical ensemble, given by

$$
\langle K(x, x ; \sigma)\rangle_{C E} \sim \frac{1}{t^{d_{S} / 2}} .
$$


In two-dimensional quantum gravity it has been established that the spectral dimension is $d_{S}=2$ while for branched polymers it has been shown to be $d_{S}=4 / 3$. This has been described in more detail in Ref. [11]. An excellent account of these equations can be found in Ref. [12, 9, 13, 14, 15].

\section{Random Matrix Model}

Every surface triangulation, $T$ has an associated dual triangulation $T^{*}$. This dual is composed of polygons connected by trivalent vertices. The matrix model formulation is such that its Feynman amplitudes are the contributions of the dual triangulation. Consider the integral over a Hermitian, $N \times N$ matrix $A=\left[a_{i j}\right]$ :

$$
\mathrm{Z}(g, N)=\int d A e^{-N\left(\frac{1}{2} \operatorname{Tr} A^{2}+g \operatorname{Tr} A^{3}\right)} \quad \text { where } \quad d A=\Pi_{i j} d \mathfrak{R} a_{i j} \Pi_{i<j} d \mathfrak{I} a_{i j} .
$$

Notice that we could instead use quadrangulations related to a non-divergent $\operatorname{Tr} A^{4}$ theory. The propagator for these theories can easily be shown to be

$$
\left\langle a_{i j} a_{k l}\right\rangle=\frac{1}{\mathrm{Z}(0, N)} \int d A a_{i j} a_{k l} e^{-\frac{N}{2} \operatorname{Tr} A^{2}}=\frac{1}{N} \delta_{i l} \delta_{j k} .
$$

A Feynman expansion of the theory partition function $F(g, N)$ has contributions of the form

$$
\frac{(N g)^{n}}{n} \int_{M} e^{-\frac{N}{2} \operatorname{Tr} A^{2}}\left(\operatorname{Tr} A^{3}\right)^{n}
$$

Each term of order $g^{n}$ in the power series expansion counts the number of diagrams with $n$ 3 -point vertices. Notice that if each triangle has area $=1$ the total area is $n$ and the weight for each vertex is $N$. So if the contribution of each Feynman diagram is determined by the above equations, each vertex $v$ contributes a factor of $N$ (cubic term), each edge $e$ (propagator) contributes a factor $N^{-1}$ and each closed loop $f$ (face: contraction of $\delta_{i i} \delta_{k k}$ in the propagator equation) contributes a factor $N$. Thus each diagram $T^{*}$ with $v(=n)$ vertices, $e$ edges and $f$ faces contributes

$$
\frac{1}{C\left(T^{*}\right)} g^{v} N^{v-e-f}=\frac{1}{C\left(T^{*}\right)} g^{v} N^{\chi},
$$

which yields the identification: $g^{v}=e^{-\beta n} N=e^{-\gamma}$, leading to the triangulation-matrix connection:

$$
Z(\Lambda, G)=\log \frac{\mathrm{Z}(g, N)}{\mathrm{Z}(0, N)} .
$$

This is the simplest case, for most quantum gravity theory realisations the inclusion of matter, the cosmological constant, more dimensions, etc, complicates the connection.

Using the gauge freedom it is possible to diagonalize the matrix $A$

$$
A \rightarrow U A U^{\dagger}=D \quad \text { where } \quad D=\operatorname{diag}\left(\lambda_{1}, \ldots, \lambda_{N}\right) .
$$

Consider the matrix model with generic action $W(A)$, it was shown in Ref. [10] that we can reduce the matrix model partition function to its eigenvalue form

$$
\mathrm{Z}=\frac{1}{N !} \int \prod_{i=1}^{N} e^{-N^{2} S_{e f f}(\lambda)} \quad \text { where } \quad S_{\text {eff }}(\lambda)=\frac{1}{N^{2}} \sum_{i=1}^{N} W\left(\lambda_{i}\right)+\frac{2}{N^{2}} \sum_{i<j} \log \left|\lambda_{i}-\lambda_{j}\right| .
$$

This equation can be interpreted as the effective potential of a system of $N$ particles in Brownian motion on an infinite straight line with coordinates given by the eigenvalues, $\lambda_{i}$. This interpretation is called the Coulomb gas model. 


\section{Project}

We are interested in finding information from the quantum gravity theory by analysing the spectral dimension associated with the matrix model in the Coulomb gas model. We can find the diffusion process associated with the Coulomb gas and its associated spectral dimension.

It is also possible to describe the geodesic distance by pure combinatorial arguments so it is interesting to find the spacetime spectral dimension purely from the matrix model formulation.

This investigation could shed some light on the relation between the spectral dimension associated with the spectra of the matrix model and the spectral dimension of spacetime.

\section{References}

[1] J. Ambjorn, J. Jurkiewicz, and R. Loll, Spectral dimension of the universe, hep-th/0505113.

[2] Z. Burda, J. D. Correia, and A. Krzywicki, Statistical ensemble of scale-free random graphs, Phys. Rev. E64 (2001) 046118, [cond-mat / 0104155$].$

[3] T. Jonsson and J. F. Wheater, The spectral dimension of the branched polymer phase of two-dimensional quantum gravity, Nucl. Phys. $\mathbf{3 5 1 5}$ (1998) 549-574, [hep-lat/9710024].

[4] J. Ambjorn, J. Jurkiewicz, and Y. Watabiki, On the fractal structure of two-dimensional quantum gravity, Nucl. Phys. B454 (1995) 313-342, [hep-lat/9507014].

[5] R. Burioni, D. Cassi, and C. Destri, Spectral partitions on infinite graphs, J. Phys. A19 (2000) 3627-3636.

[6] R. Loll, Discrete aproaches to quantum gravity in four dimensions, Living Rev. Relativity 1 (1998) [http://www.livingreviews.org/lrr-1998-13].

[7] K. Schleich and D. Witt, Numerical quantum gravity by dynamical triangulation, gr-qc/9612018.

[8] J. Ambjorn, J. Jurkiewicz, and R. Loll, Dynamically triangulating lorentzian quantum gravity, Nucl. Phys. B610 (2001) 347-382, [hep-th/ 0105267 ].

[9] T. Jonsson and J. F. Wheater, The spectral dimension on branched polymer ensembles, . Prepared for NATO Advanced Research Workshop on Theoretical Physics: New Developments in Quantum Field Theory, Zakopane, Poland, 14-20 Jun 1997.

[10] E. Brezin, C. Itzykson, G. Parisi, and J. B. Zuber, Planar diagrams, Commun. Math. Phys. 59 (1978) 35.

[11] J. D. Correia and J. F. Wheater, The spectral dimension of non-generic branched polymer ensembles, Phys. Lett. B422 (1998) 76-81, [hep-th/9712058].

[12] J. Ambjorn, D. Boulatov, J. L. Nielsen, J. Rolf, and Y. Watabiki, The spectral dimension of $2 d$ quantum gravity, JHEP 02 (1998) 010, [hep-th/ 9801099 ].

[13] J. Ambjorn, Quantization of geometry, hep-th/9411179.

[14] J. Ambjorn, K. N. Anagnostopoulos, T. Ichihara, L. Jensen, and Y. Watabiki, Quantum geometry and diffusion, JHEP 11 (1998) 022, [hep-lat/9808027].

[15] J. Ambjorn, B. Durhuus, and T. Jonsson, Quantum geometry. a statistical field theory approach, Cambridge Monogr. Math. Phys. 1 (1997) 1-363. 